\title{
A phase II study of radioimmunotherapy with intraventricular 131|-3F8 for medulloblastoma
}

Kim Kramer ${ }^{1}$, Neeta Pandit-Taskar ${ }^{2}$, John L. Humm², Pat B. Zanzonico ${ }^{2}$, Sofia Haque ${ }^{2}$, Ira J. Dunkel ${ }^{1}$, Suzanne L. Wolden ${ }^{3}$, Maria Donzelli ${ }^{1}$, Debra A. Goldman ${ }^{4}$, Jason S. Lewis ${ }^{2}$, Serge K. Lyashchenko ${ }^{2}$, Yasmin Khakoo ${ }^{1}$, Jorge A. Carrasquillo², Mark M. Souweidane ${ }^{5}$, Jeffrey P. Greenfield ${ }^{5}$, David Lyden ${ }^{1}$, Kevin D. De Braganca ${ }^{1}$, Stephen W. Gilheeney ${ }^{1}$, Steven M. Larson ${ }^{2}$, Nai-Kong V. Cheung ${ }^{1}$

${ }^{1}$ Department of Pediatrics, Memorial Sloan Kettering Cancer Center, New York

${ }^{2}$ Department of Radiology (Molecular Imaging and Therapy Service), Memorial Sloan Kettering Cancer Center, New York

${ }^{3}$ Department of Radiation Oncology, Memorial Sloan Kettering Cancer Center, New York

${ }^{4}$ Department of Epidemiology \& Biostatistics, Weill Cornell Medical College, New York

${ }^{5}$ Department of Neurosurgery, Weill Cornell Medical College, New York

\section{Abstract}

Background: High-risk and recurrent medulloblastoma (MB) is associated with significant mortality. The murine monoclonal antibody 3F8 targets the cell-surface disialoganglioside GD2 on MB. We tested the efficacy, toxicity, and dosimetry of compartmental radioimmunotherapy (cRIT) with intraventricular ${ }^{131}$ I-labeled $3 \mathrm{~F} 8$ in patients with $\mathrm{MB}$ on a phase II clinical trial.

Methods: Patients with histopathologically confirmed high-risk or recurrent MB were eligible for cRIT. After determining adequate cerebrospinal fluid (CSF) flow, patients received $2 \mathrm{mCi}$ (where $\mathrm{Ci}$ is Curie) ${ }^{124} \mathrm{I}-3 \mathrm{~F} 8$ or ${ }^{131} \mathrm{I}-3 \mathrm{~F} 8$ with nuclear imaging for dosimetry, followed by up to four therapeutic ( $10 \mathrm{mCi} / \mathrm{dose}){ }^{131} \mathrm{I}-3 \mathrm{~F} 8$ injections. Dosimetry estimates were based on serial CSF and blood samplings over $48 \mathrm{hr}$ plus region-of-interest analyses on serial imaging scans. Disease evaluation included pre- and posttherapy brain/spine magnetic resonance imaging approximately every 3 months for the first year after treatment, and every 6-12 months thereafter.

Results: Forty-three patients received a total of 167 injections; 42 patients were evaluable for outcome. No treatment-related deaths occurred. Toxicities related to drug administration included acute bradycardia with somnolence, headache, fatigue, and CSF pleocytosis consistent with chemical meningitis and dystonic reaction. Total CSF absorbed dose was 1,453 cGy (where Gy is Gray; 350.0-2,784). Median overall survival from first dose of cRIT was 24.9 months (95\% confidence interval [CI]:16.3-55.8). Patients treated in radiographic and cytologic remission were

Correspondence: Kim Kramer, Department of Pediatrics, Memorial Sloan Kettering Cancer Center, 1275 York Ave, Box 429, New York, NY 10065., kramerk@mskcc.org.

SUPPORTING INFORMATION

Additional Supporting Information may be found online in the supporting information tab for this article. 
at a lower risk of death compared to patients with radiographically measurable disease (hazard ratio: $0.40,95 \%$ CI: $0.18-0.88, P=0.024)$.

Conclusions: cRIT with ${ }^{131} \mathrm{I}-3 \mathrm{~F} 8$ is safe, has favorable dosimetry to CSF, and when added to salvage therapy using conventional modalities, may have clinical utility in maintaining remission in high-risk or recurrent MB.

\section{Keywords}

CNS tumors; intrathecal therapy; medulloblastoma; radioimmunotherapy; 3F8

\section{1| INTRODUCTION}

Few curative treatment options exist for recurrent medulloblastoma (MB). Some studies support a role for high-dose chemotherapy with autologous hematopoietic cell rescue, although cure for patients with previously irradiated recurrent MB is unlikely. ${ }^{1-3}$ Direct intrathecal delivery of radiolabeled tumor-specific antibodies or peptides may aid in both detection and treatment of recurrent leptomeningeal disease. ${ }^{4-11}$

We previously described a murine monoclonal IgG3 antibody, 3F8, that recognizes disialoganglioside GD2, a tumor antigen homogeneously distributed on the cell membrane of solid tumors of neuroectodermal origin, including MB. ${ }^{12-14}$ Gangliosides are cell membrane associated lipid-sugar compounds thought to influence a variety of cellular functions including those affecting tumorigenesis. 3F8 is nonreactive with most normal human tissues including bone marrow, colon, stomach, heart, lung, muscle, thyroid, testes, pancreas; expression is found on central neurons and peripheral nerves. Intravenous antiGD2 therapy is standard of care for patients with metastatic neuroblastoma. ${ }^{15,16} 3 \mathrm{~F} 8$ radiolabeled with I-124 and I-131 retains its immunoreactive properties. ${ }^{17,18}$ Improvement in overall survival (OS) has been noted with the incorporation of compartmental intraventricular radioimmunotherapy (cRIT) including ${ }^{131}$ I-labeled $3 \mathrm{~F} 8$ for patients with relapsed central nervous system neuroblastoma. ${ }^{19}$

We evaluated the efficacy, dosimetry, and toxicity of cRIT with ${ }^{124} \mathrm{I}$ - and ${ }^{131}$ I-labeled 3F8 via Ommaya catheters in patients with high-risk ( $<3$ years of age with nondesmoplastic histology and no prior radiation therapy or with refractory M3 disease) or recurrent MB/ primitive neuroectodermal tumor (PNET) on a phase II clinical trial at Memorial Sloan Kettering Cancer Center (MSK) between 2006 and 2016.

\section{2 | PATIENTS AND METHODS}

Study NCT00445965 is an open study available for adult and pediatric patients with GD2expressing CNS malignancies including MB. The primary aim of this study was to determine the $\mathrm{OS}$ of patients with MB following intra-Ommaya ${ }^{131} \mathrm{I}-3 \mathrm{~F} 8$, to determine the response rate to ${ }^{131} \mathrm{I}-3 \mathrm{~F} 8$ in this population, and secondarily, to determine the cumulative toxicities of serial injections of intra-Ommaya ${ }^{131} \mathrm{I}-3 \mathrm{~F} 8$. 


\subsection{F8 monoclonal antibody (MoAb)}

MoAb 3F8 was purified at MSK. ${ }^{124} \mathrm{I}-3 \mathrm{~F} 8$ and ${ }^{131} \mathrm{I}-3 \mathrm{~F} 8$ radiolabeling and dose preparation were performed at the MSK Radiochemistry and Molecular Imaging Probe Core. For every patient dose, $2 \mathrm{mg}$ of 3F8 were radiolabeled using the iodogen method. Every batch was quality control tested to assure conformance to the acceptance specifications detailed in an US Food and Drug Administration (FDA) acknowledged investigative new drug application. The total mass of 3F8 in both $2 \mathrm{mCi}$ (where $\mathrm{Ci}$ is Curie) dosimetry doses and $10 \mathrm{mCi}$ therapy doses was adjusted to approximately $2 \mathrm{mg}$ with cold $3 \mathrm{~F} 8 .^{12,17,18}$

\subsection{Eligibility}

The subject of this analysis includes all patients with a histologically confirmed diagnosis of high-risk or recurrent MB or PNET. High-risk patients were those less than 3 years of age at diagnosis with non-desmoplastic histology and no history of radiation therapy or those with refractory $\mathrm{M}+$ disease following radiation and chemotherapy Patients with high-risk disease who had residual disease after initial surgery, radiation therapy, and chemotherapy were also eligible for cRIT upfront prior to disease progression.

All other patients had recurrent MB following standard therapy. Molecular grouping by WNT, SHH, or CMYC status was generally not performed. Patients demonstrated a normal or less than grade 3 kidney and liver profile, platelets $>50,000 / \mu \mathrm{l}$ and absolute neutrophil count $>1,000 / \mu \mathrm{l}$. Prior focal or craniospinal radiation therapy was permitted, but not within 3 weeks prior to $3 \mathrm{~F} 8$ injections. Prior radiation limits were confined to less than $72 \mathrm{~Gy}$ (where Gy is Gray) focal brain or 45 Gy craniospinal radiation therapy. Adequate cerebrospinal fluid (CSF) flow was confirmed by a pretreatment intra-Ommaya Indium-111 diethylenetriaminepentaacetic acid biodistribution study. Patients with obstructive or symptomatic communicating hydrocephalus were excluded. The protocol (MSK 05-122, NCT00445965) was approved by the MSK Institutional Review Board (IRB) and performed under an FDA-approved investigational new drug. Informed consent was obtained in accordance with MSK IRB policy

\section{3 | cRIT therapy}

Pretreatment evaluation included a detailed history, physical examination, complete blood count, metabolic profile, liver function, and thyroid function tests. CSF from the indwelling Ommaya catheter was examined for total protein, glucose, cell count, and cytology. Patients had a magnetic resonance imaging (MRI) of the brain and spine with and without gadolinium within 3 weeks prior to the dosimetry injection. To minimize the uptake of radioiodine by the thyroid, patients were premedicated with oral saturated solution of potassium iodide drops and liothyronine starting 5-7 days prior to the first injection and continued for 2 weeks after the last injection. To prevent a possible allergic or meningitic reaction, patients received oral acetaminophen and diphenhydramine prior to each injection. Oral or intravenous dexamethasone $0.5-1 \mathrm{mg}$ (twice daily for a total of six doses) was given with each injection. The dexamethasone was a flat dose (older patients or $>20 \mathrm{~kg}$ received 1 $\mathrm{mg}$ ). Ceftriaxone was given approximately $1 \mathrm{hr}$ after each injection. 
All patients received an initial dosimetry dose $\left(2 \mathrm{mCi}\right.$ and $2 \mathrm{mg}$ total MoAb) ${ }^{124} \mathrm{I}-3 \mathrm{~F} 8$ or ${ }^{131} \mathrm{I}-3 \mathrm{~F} 8$ in week 1 , followed by a weekly $10 \mathrm{mCi}(2 \mathrm{mg}){ }^{131} \mathrm{I}-3 \mathrm{~F} 8$ therapy injection up to four injections. All injections were performed with a syringe fitted with a $0.22 \mu \mathrm{m}$ filter. In the first cohort of patients, dosimetry was assessed by Ommaya CSF and blood sampling and serial ${ }^{124} \mathrm{I}-3 \mathrm{~F} 8 \mathrm{PET} / \mathrm{CT}$ (where PET is positron emission tomography) through $48 \mathrm{hr}$; the trial was later changed to dosimetry using CSF sampling and serial ${ }^{131} \mathrm{I}-3 \mathrm{~F} 8$ singlephoton emission computed tomography (SPECT) through $48 \mathrm{hr}$ (Figs. 1A and 1B). Toxicity was defined by the Common Terminology Criteria for Adverse Events Version 3.0. Patients had follow-up on the second and third day after the dosimetry injection, and then once per week during the therapy injection period. After completing cRIT injections, patients were evaluated via history, physical examination, serology, CSF cytology, MRI brain, and spine approximately 1 month later.

2.3.1 Evaluation of response-Conventional MRI brain imaging included sagittal and axial T1-weighted, axial T2-weighted, axial fluid-attenuated inversion recovery (FLAIR), axial diffusion weighted images, apparent diffusion coefficient and exponential, and postcontrast T1-weighted sequences in axial, sagittal, and coronal planes. In particular, diffusion restriction, in conjunction with enhancement on postcontrast images, indicated MB as they are hypercellular and therefore diffusion restrict.

At the time of study entry, tumor size was evaluated on contrast-enhanced MRI brain and spine with gadolinium. Any patient with disease seen on contrast-enhanced MRI of the brain or spine, or with extrinsic cells in the CSF cytology obtained by ventricular sampling, was considered to have evaluable disease. Patients were considered to be in remission at the time of cRIT, if CSF cytology by ventricular testing was negative for malignant cells and if radiographically identifiable tumor was absent on comparison scans. A complete response post-therapy was defined as the absence of all identified disease utilizing MRI and/or clearance of CSF cytology. A partial response was defined at least 50\% decrease in tumor size demonstrable by MRI or decrease in leptomeningeal enhancement; stable disease (SD) was defined as less than $50 \%$ decrease or no reduction in tumor size demonstrable by MRI, or tumor growth that is less than the criteria defined as progressive disease. Progressive disease was defined as the disease in previously uninvolved areas or clinical or radiological evidence of increased volume of greater than $25 \%$ in tumor area with maximum perpendicular diameters in any site of residual disease compared to immediate prestudy area, or increased leptomeningeal enhancement on MRI. Brain and spine MRI were performed approximately 1 month after the last cRIT 3F8 injection and approximately every 3 months for the first year, and every 6-12 months annually thereafter. OS and progression-free survival (PFS) were determined using the Kaplan-Meier method whereby OS was calculated from the date of diagnosis or recurrence until death or last follow-up. OS from the time of first cRIT injection until death or last follow-up was also calculated. It is quite possible that ${ }^{131} \mathrm{I}-3 \mathrm{~F} 8$ may lead to disease stabilization without complete or partial responses in this population with advanced disease. We therefore propose OS at 6 months as an endpoint of this study. Patients receiving ${ }^{131} \mathrm{I}-3 \mathrm{~F} 8$ on this trial have typically completed standard initial and subsequent salvage chemotherapy. OS at 6 months was the primary endpoint for this study. The study contained all histologies, while the analysis presented hereincludes only the 
patients with MB. Therefore, no power analysis was planned in advance for this component of the study.

2.3.2 Dosimetry-Dosimetry to the CSF in the ventricles and within the thecal sac was determined by region-of-interest analysis ${ }^{124} \mathrm{I}-3 \mathrm{~F} 8 / \mathrm{PET}$ imaging or ${ }^{131} \mathrm{I}-3 \mathrm{~F} 8 / \mathrm{SPECT}$ gamma camera imaging obtained at approximately 4,24 , and $48 \mathrm{hr}$ after injection of a $2 \mathrm{mCi}$ administered activity as previously described. ${ }^{4}{ }^{124} \mathrm{I}-3 \mathrm{~F} 8 / \mathrm{PET}$ was the preferred tracer but both availability and expense of ${ }^{124} \mathrm{I}-3 \mathrm{~F} 8 / \mathrm{PET}$ precluded its regular use, where ${ }^{131} \mathrm{I}-3 \mathrm{~F} 8 /$ SPECT was always available at a significantly reduced cost. CSF and blood dosimetry was also concurrently estimated by corresponding samples obtained at the same time points postinjection and measured aliquots were radioassayed in a scintillation well counter calibrated for ${ }^{124} \mathrm{I}$ or ${ }^{131} \mathrm{I}$.The pharmacokinetic data obtained from PET orgamma-camera imaging and CSF sampling were combined with anatomical imaging information to estimate the absorbed dose to brain, spinal cord, and blood as previously described. ${ }^{20-22}$ Given the risk of radionecrosis in the setting of prior conventional craniospinal radiation therapy with cRIT was unknown, a conservative estimate for cumulative dosing was used, recognizing the general spinal cord tolerance limit is $45 \mathrm{~Gy}$. To avoid the risk of radiation toxicity in patients who had prior craniospinal radiation, the administered activity (dose) was reduced to ensure that the CSF radiation dose delivered by ${ }^{131} \mathrm{I}-3 \mathrm{~F} 8$ did not exceed $2,400 \mathrm{cGy}$ as determined by CSF sampling and serial nuclear medicine scans. In such patients, the maximum cumulative dose allowed by cRIT therapy injections was $2,400 \mathrm{cGy}$ to the CSF, thereby requiring a dose adjustment to avoid exceeding this maximum.

Patient and treatment characteristics were described using frequencies and percentage for categorical variables, and medians and ranges for continuous variables. OS was calculated from the time of first I-3F8 injection until death. Patients alive at last follow-up were censored. PFS was calculated from the time of first I-3F8 injection until progression or death. Patients alive and disease-free at last follow-up were censored. OS and PFS were graphically displayed using Kaplan-Meier plots, and median, 6-month, 1-year, and 5-year Kaplan-Meier survival estimates were provided. The relationship between OS and patient characteristics was assessed with univariate Cox proportional hazards regression. $P$-values less than 0.05 were considered statistically significant, and all analyses were performed using SAS 9.4 (The SAS Institute, Cary, NC).

\section{3 | RESULTS}

Forty-three patients with high-risk $(\mathrm{N}=8)$ and recurrent $(\mathrm{N}=35) \mathrm{MB}$ received 167 injections cRIT ${ }^{131}$ I-3F8 (124 therapy injections; 43 dosimetry injections). With limitations in patients who received prior craniospinal radiation therapy, 18 patients received 4 fulltherapy doses as planned, 13 patients received 3, 6 patients received 2, and 6 patients received 1 full-therapy dose. One patient was removed following the dosimetry dose due to clinical and radiographic progression of disease; he did not receive any subsequent therapy injections. 
In total, 42 patients were evaluable. Patient demographics are presented in Table 1. Changing stages prior to cRIT included M0 $(n=2)$, M2 $(n=5), M 3(n=33)$, and M3/M4 (n $=2$ ).

\section{1| Response}

One patient was removed from the study because of clinical deterioration prior to ever receiving a therapy injection of ${ }^{131} \mathrm{I}-3 \mathrm{~F} 8$. Of 22 patients starting cRIT with radiographically evaluable disease on MR, 20 had a CSF cytology positive for MB at some point in the course of treatment. Of these 22, MRI brain and spine remained stable in 9 patients; 1 patient had improvement/near resolution of leptomeningeal enhancement, and $12 \mathrm{had}$ progression of disease, including 3 with progressing bulky disease when starting cRIT. Of the remaining 20 patients who started cRIT with no evaluable radiographic or cytologic disease, 15 remained free of disease post-cRIT; 5 had progression of disease.

\section{2 | Survival}

By the end of follow-up, 27 patients had died with a median OS of 24.9 months (95\% confidence interval [CI]: 16.3-55.8 months). Six-month, 1-year, and 5-year OS estimates were $88.1 \%$ (95\% CI: 73.7-94.9\%), 78.6\% (95\% CI: 62.9-88.2\%), and $44.9 \%$ (95\% CI: 29.0-59.5\%), respectively (Fig. 2A). Median PFS was 11 months (95\% CI: 2.0-16.8 months); 11 patients were alive and progression-free at the end of follow-up. Six-month, 1year, and 5-year PFS estimates were 57.1\% (95\% CI: 40.9-70.4\%), 47.6\% (95\% CI: 32.1$61.6 \%$ ), and $23.7 \%$ (95\% CI: 11.7-38.0\%), respectively (Fig. 2B).

There was one death in a long-term survivor from second malignancy, an 8-year-old male who died of secondary glioblastoma multiforme 5.5 years after initial MB diagnosis. Another 5-year-old female achieved CNS remission for 1-year postrelapse, but died of extraneural MB 1.5 years later.

Of the 15 patients alive, 12 remain well off all therapy, while 2 have received maintenance therapy including oral etoposide, oral Temodar with irinotecan, intravenous bevacizumab, and intrathecal arabinoside; one patient has been receiving therapy for extraneural metastases. Characteristics of long-term survivors can be found in Table 2 .

Age (hazard ratio [HR]: $1.03,95 \%$ CI: $0.98-1.07, P=0.22$ ) or having prior irradiation (HR: $2.00,95 \%$ CI: $0.47-8.49, P=0.35)$ was not significantly associated with OS. Anaplastic histology was marginally associated with OS where patients with anaplastic histology were at a higher risk of death (HR: $2.29,95 \%$ CI: $0.96-5.46, P=0.06$ ). Status at the time of cRIT was associated with OS where patients in remission were at a lower risk of death compared to patients with radiographically measurable disease (HR: $0.39,95 \%$ CI: $0.18-0.88, P=$ 0.024). No association was found between CSF dose and OS (HR: 1.00, 95\% CI: 1.00-1.01, $P=0.29)$.

\subsection{Acute side effects of CRIT ${ }^{131}$ I-3F8}

A secondary aim of this study was assessment of acute and long-term toxicities. cRIT 3F8 was routinely administered in the outpatient setting, with supportive care provided 
throughout the day of each injection. Patients were treated awake at the bedside. There were no treatment-related deaths during the injection period or postinjection observation period. Adverse events were self-limited, occurring in the first several hours after injection, and most commonly after the first (dosimetry) injection. This injection was commonly associated with grade 2 or 3 fever, headache, nausea, and vomiting; symptoms were less commonly observed with subsequent injections. In addition to these anticipated toxicities, other unexpected grade 3 but reversible adverse events possibly or definitely related to drug administration included transient acute bradycardia with somnolence requiring inpatient observation ( $\mathrm{N}=2)$, headache, fatigue, and CSF pleocytosis consistent with chemical meningitis treated with corticosteroids $(\mathrm{N}=1)$, and acute dystonic reaction treated with both benzodiazepine and antihistamine $(\mathrm{N}=1)$. The administered activities of cRIT ${ }^{131} \mathrm{I}-3 \mathrm{~F} 8$ therapy injections were primarily limited by the maximum allowed by protocol, ${ }^{4}$ or by dosimetry estimates such that 2,400 cGy to the CSF was delivered for patients with prior craniospinal radiation therapy There were no complications related to raised intracranial pressure, hemorrhage, or infection.

\section{4 | Long-term toxicities}

There were no long-term toxicities directly attributed to cRIT 3F8. We specifically note the absence of radionecrosis in any patient in this cohort, despite receiving prior conventional radiation therapy, and additional radiation therapy delivered by cRIT. However, among longterm survivors with a history of prior high-dose induction chemotherapy, +/- myeloablative regimens, craniospinal radiation therapy, and cRIT 3F8, toxicity included symptomatic Moyamoya syndrome $(\mathrm{N}=1)$ and symptomatic small-vessel angiopathy $(\mathrm{N}=1)$. One patient with Gorlin syndrome developed a meningioma, ovarian fibrothecoma, and basal cell carcinoma, all developing more than 6 years after cRIT.

\subsection{Dosimetry}

All patients had distribution of the cRIT 3F8 demonstrated throughout the thecal sac on serial nuclear scans through 48-hr postdosimetry injection, although some irregularity in distribution around cerebral convexities was seen in some patients. By CSF sampling, the median dose to the CSF was $47.9 \mathrm{cGy} / \mathrm{mCi}(14.8-207.6)$ and to the blood $1.6 \mathrm{cGy} / \mathrm{mCi}(0.1-$ 9.2). The median total dose delivered to the CSF was $1,453 \mathrm{cGy}(350.0-2,784)$. The dosimetry estimations with ${ }^{124} \mathrm{I}-3 \mathrm{~F} 8$ PET scans were generally similar to those of CSF sampling, while that with ${ }^{131} \mathrm{I}-3 \mathrm{~F} 8$ gamma camera imaging were generally higher, more so for ventricular doses than for spinal canal region (Table 3). The protocol mandated CSF and blood sampling only after the first dose, but to see how reproducible dosimetry estimates were, there were nine patients who had additional blood and CSF tested after each therapy injection.

Among nine patients who had blood and CSF samples analyzed for all four ${ }^{131} \mathrm{I}-3 \mathrm{~F} 8 \mathrm{cRIT}$ therapies, there was large variability for both blood (\%SD = 30-169\%) and CSF (\%SD = 15-98\%) dose estimates with sampling (Supplementary Table S1). It was observed that dosimetry did not seem to be dependent on disease status. 


\section{4 | DISCUSSION}

cRIT with intraventricular ${ }^{131} \mathrm{I}-3 \mathrm{~F} 8$ appears safe with manageable acute adverse events, even in a very heavily pretreated patient population. Injections were routinely given in the outpatient setting, with supportive care treating anticipated acute toxicities. Although the dose-limiting toxicity for most radioimmunotherapy trials is myelosuppression, the dosing employed in this study was not associated with severe myelosuppression. Antitumor effect is attributed to the direct effect of beta plus gamma irradiation from ${ }^{131} \mathrm{I}$-emission, although it is plausible that antitumor cytotoxicity may be in part due to complement activation within the CSF space. Previous observations in patients treated with rituximab suggested the activation of complement $\mathrm{C} 3$ and $\mathrm{C} 5 \mathrm{~b}-9$ in the $\mathrm{CSF}$ after intraventricular administration for recurrent CNS lymphoma. ${ }^{23}$

Although preliminary data have shown that some young children with MB may be cured with chemotherapy alone, the poor survival results for patients with recurrent MB have been noted in many series. ${ }^{1-3,24-28}$ Given experience with targeted immunotherapy efficacy when used as consolidation in the setting of minimal residual disease, this trial allowed for patients to enter cRIT without radiographically evaluable disease. It is acknowledged, however, that because of this, it is not appropriate to compare efficacy directly to other studies that mandated evaluable disease. However, given the extended survival for this cohort of patients, which includes patients with high-risk disease that still had evidence of leptomeningeal tumor after upfront chemotherapy and radiation therapy, cRIT ${ }^{131} \mathrm{I}-3 \mathrm{~F} 8$ is encouraging, particularly when noting the progression-free and OS rates for patients with relapsed MB in other series published to date (Table 4). Status at the time of cRIT was related to OS, where patients in near radiographic and cytologic remission were at a lower risk of death compared to patients with progressive or radiographically measurable disease. It is important to note that we did not find an association between dose and OS. This would suggest that current cRIT ${ }^{131} \mathrm{I}-3 \mathrm{~F} 8$ dosing is adequate to improve survival. Eleven of 15 long-term survivors received cRIT in the setting of radiographic and cytologic remission and remained in radiographic and cytologic remission; the other 4 patients had minimal and unchanged persistent focal nodularity on MRI. However, at the $10 \mathrm{mCi}$ therapy dose, there does not appear to be a benefit of GD2-directed cRIT 3F8 in the setting of bulky progressive MB. Given that the maximum CSF dose allowed on this study for patients with prior craniospinal radiation therapy was $2,400 \mathrm{cGy}$, it is possible that increasing the number of injections and total dose to the CSF may contribute to disease control for patients with mea- sureable disease. Importantly, given the absence of radionecrosis in this study cohort, a dose escalation should be considered in future studies. ${ }^{29}$ Although this was not a randomized comparison between second remission consolidation with or without cRIT, the prolonged survival for patients receiving cRIT suggest a survival advantage compared to historical estimates. $^{24}$

An important aim of this study was to determine the doses delivered to the CSF by cRIT, with an understanding that the dose to tumor cells binding antibody can be many times higher than the average dose estimates to the CSF. ${ }^{20,21,30} \mathrm{CSF}$ dosimetry was performed using two independent methods. The first used direct measurement of the activity concentrations derived from serial CSF samples removed from the ventricular reservoir; the 
second used quantitative serial gamma camera images acquired at three nominal time points. CSF samples obtained from the Ommaya reservoir may not be representative of CSF in other areas of the thecal space. In contrast, the gamma camera method determines counts from the entire CSF, where calibration of gamma camera counts into units of activity is critical. While dose estimates from the CSF sampling correlated with those from the gamma camera method for some patients, others were discrepant. Of note was the fairly consistent dose to blood, a surrogate for red marrow in all patients, while the CSF cGy/mCi dose was variable. This is likely a reflection of variable rates of CSF flow based on prior surgery, radiation, presence or absence of bulk lesions, all leading to inherent CSF flow differences prior to cRIT. As CSF volumes were corrected for age as is standard for intrathecal drug delivery, it is not anticipated that CSF volume/body size of the child impacted CSF dose estimates. However, a high therapeutic index of tumor to blood was achieved in all patients. In general, the dosimetry obtained by serial ${ }^{124} \mathrm{I}-3 \mathrm{~F} 8$ PET scans provided the most precise estimates of cGy delivered to various sites including the ventricles, cervical, thoracic, and lumbosacral spine; as a less precise imaging modality, dosimetry-based ${ }^{131}$ I-3F8 PET (Figs. $1 \mathrm{~A}$ and $1 \mathrm{~B}$ ) consistently yielded higher ventricular dose than either CSF sampling or PET imaging. The choice of dosimetry method could affect treatment planning.

Further studies may incorporate an analysis of GD2 expression with response and survival. In addition, patients with both classic MB and PNET were eligible for this study, so while recent data suggest that the tumors are not as related as once thought, all patients were included in this analysis. Moving forward, the distinction between MB and PNET will be made. Additional efforts are being made to subgroup patients prospectively, but at the time this trial accrued patients, this molecular subgroup analysis was not routinely undertaken. In the present era of molecular profiling of MB into distinct subtypes with prognostic implications, it is now possible to consider dose reduction of therapies with known neurocognitive risks such as CSI. The findings presented suggest that prolonged survival with cRIT treatment is possible in a subset of patients. Finally, we note that although this study used a MoAb targeting GD2, other tumor-associated antigens may similarly be exploited; we have developed another antibody $8 \mathrm{H} 9$ targeting $\mathrm{B} 7-\mathrm{H} 3$, also found to be highly overexpressed on MB and may also be considered for cRIT. ${ }^{31}$

\section{5| CONCLUSIONS}

Intraventricular radioimmunotherapy can be successfully incorporated into treatment strategies as consolidation for relapsed and high-risk MB. The role of cRIT in patients with bulky tumors at the current dosing levels is questionable. Future studies are warranted to evaluate feasibility of higher dosing or differentiate treatment strategies in those with bulky disease.

\section{Supplementary Material}

Refer to Web version on PubMed Central for supplementary material. 


\title{
ACKNOWLEDGMENTS
}

\begin{abstract}
We are grateful for the expertise and support from Richard O'Reilly, MD; and Jonathan Finlay, MD; Jing Qiao and Jiong Wufrom the Radiochemistry Laboratory; Amabella Lindo and Louise Harris from the Nuclear Medicine team; Christopher Horan, Matthew Williamson, and Lawrence T. Dauer, $\mathrm{PhD}$, from the Department of Radiation Safety; Ursula Tomlinson, PNP, Ester Dantis, FNP, Cheryl Fischer, PNP, and Mary Petriccione, PNP, from the Pediatric Oncology Team; Karima Yataghene, MD, Elizabeth Chamberlain, Samantha Leyco, and Esther Kwak from the Research Study Team; and Joseph Olechnowicz for editorial assistance. We also acknowledge the exceptional expertise of the MSK Pediatric Nursing staff for clinical supervision of the patients. This study was supported in part by the National Cancer Institute grants K08 CA072868, the Robert Steel Foundation, Catie Hoch Foundation, Katie's Find A Cure Fund, Pediatric Cancer Foundation and the Leptomeningeal Research Fund. We also acknowledge the National Cancer Institute Cancer Center Support Grant/Core Grant (P30 CA008748).

Funding information
\end{abstract}

Grant sponsor: National Cancer Institute; Grant number: K08 CA072868; Grant sponsor: Robert Steel Foundation; Grant sponsor: Catie Hoch Foundation; Grant sponsor: Katie's Find A Cure Fund; Grant sponsor: Pediatric Cancer Foundation; Grant sponsor: Leptomeningeal Research Fund; Grant number: P30 CA008748

\section{CONFLICT OF INTEREST}

Antibody 3F8 was licensed to Ymabs, Inc., by MSKCC. MSKCC and NKC have financial interest in Ymabs.

\author{
Abbreviations: \\ cRIT \\ $\mathbf{C i}$ \\ CI \\ CSF \\ FDA \\ Gy \\ HR \\ IRB \\ MB \\ MoAb \\ MRI \\ MSK \\ OS \\ PET \\ PFS \\ PNET \\ SPECT \\ compartmental radioimmunotherapy \\ Curie \\ confidence interval \\ cerebrospinal fluid \\ US Food and Drug Administration \\ Gray \\ hazard ratio \\ Institutional Review Board \\ medulloblastoma \\ monoclonal antibody \\ magnetic resonance imaging \\ Memorial Sloan Kettering Cancer Center \\ overall survival \\ positron emission tomography \\ progression-free survival \\ primitive neuroectodermal tumor \\ single-photon emission computed tomography
}




\section{REFERENCES}

1. Egan G, Cervone KA, Philips PC, et al. Phase I study of temozolomide in combination with thiotepa and carboplatin with autologous hematopoietic cell rescue in patients with malignant brain tumors with minimal residual disease. Bone Marrow Transplant. 2016;51(4):542-545. [PubMed: 26726947]

2. Dunkel IJ, Boyett JM, Yates A, et al. High-dose carboplatin, thiotepa, and etoposide with autologous stem-cell rescue for patients with recurrent medulloblastoma. Children's Cancer Group. J Clin Oncol. 1998;16(1):222-228. [PubMed: 9440746]

3. Gururangan S, Krauser J, Watral MA, et al. Efficacy of high-dose chemotherapy or standard salvage therapy in patients with recurrent medulloblastoma. Neuro-Oncology. 2008;10(5):745-751. [PubMed: 18755919]

4. Kramer K, Humm JL, Souweidane MM, et al. Phase I study of targeted radioimmunotherapy for leptomeningeal cancers using intra-Ommaya 131-I-3F8. J Clin Oncol. 2007;25(34):5465-5470. [PubMed: 18048828]

5. Moseley RP, Papanastassiou V, Zalutsky MR, et al. Immunoreactivity, pharmacokinetics and bone marrow dosimetry of intrathecal radioim- munoconjugates. Int J Cancer. 1992;52(1):38-43. [PubMed: 1500225]

6. Pizer BL, Papanastassiou V, Moseley R, et al. Meningeal leukemia and medulloblastomapreliminary experience with intrathecal radioimmunotherapy. Antibody Immunoconj. 1991;4(4): 753-761.

7. Papanastassiou V, Pizer BL, Coakham HB, et al. Treatment of recurrent and cystic malignant gliomas by a single intracavity injection of 131I monoclonal antibody: feasibility, pharmacokinetics and dosimetry. BrJ Cancer. 1993;67(1):144-151. [PubMed: 8427774]

8. Bigner DD, Brown MT, Friedman AH, et al. Iodine-131-labeled antitenascin monoclonal antibody 81C6 treatment of patients with recurrent malignant gliomas: phase I trial results. J Clin Oncol. 1998;16(6):2202-2212. [PubMed: 9626222]

9. Cokgor I, Akabani G, Friedman HS, et al. Long term response in a patient with neoplastic meningitis secondary to melanoma treated with (131)I-radiolabeled antichondroitin proteoglycan sulfate Mel-14 F(ab')(2): a case study. Cancer. 2001;91(9):1809-1813. [PubMed: 11335907]

10. Zalutsky MR, Boskovitz A, Kuan CT, et al. Radioimmunotargeting of malignant glioma by monoclonal antibody D2C7 reactive against both wild-type and variant III mutant epidermal growth factor receptors. Nucl Med Biol. 2012;39(1):23-34. [PubMed: 21958852]

11. Reardon DA, Zalutsky MR, Akabani G, et al. A pilot study: 131I- antitenascin monoclonal antibody 81c6 to deliver a 44-Gy resection cavity boost. Neuro-oncology. 2008;10(2):182-189. [PubMed: 18287339]

12. Cheung NK, Landmeier B, Neely J, et al. Complete tumor ablation with iodine 131-radiolabeled disialoganglioside GD2-specific monoclonal antibody against human neuroblastoma xenografted in nude mice. J Natl Cancer Inst. 1986;77(3):739-745. [PubMed: 3091900]

13. Dobrenkov K, Cheung NK. GD2-targeted immunotherapy and radioimmunotherapy. Semin Oncol. 2014;41(5):589-612. [PubMed: 25440605]

14. Mennel HD, Bosslet K, Geissel H, et al. Immunohistochemically visualized localisation of gangliosides Glac2 (GD3) and Gtri2 (GD2) in cells of human intracranial tumors. Exp Toxicol Pathol. 2000;52(4):277-285. [PubMed: 10987178]

15. Yu AL, Gilman AL, Ozkaynak MF, et al. Anti-GD2 antibody with GM-CSF, interleukin-2, and isotretinoin for neuroblastoma. New Engl J Med. 2010;363(14):1324-1334. [PubMed: 20879881]

16. Cheung NK, Cheung IY, Kushner BH, et al. Murine anti-GD2 monoclonal antibody 3F8 combined with granulocyte-macrophage colony-stimulating factor and 13-cis-retinoic acid in high-risk patients with stage 4 neuroblastoma in first remission. J Clin Oncol. 2012;30(26):3264-3270. [PubMed: 22869886]

17. Larson SM, Pentlow KS, Volkow ND, et al. PET scanning of iodine-124-3F9 as an approach to tumor dosimetry during treatment planning for radioimmunotherapy in a child with neuroblastoma. J Nucl Med. 1992;33(11):2020-2023. [PubMed: 1432165] 
18. Yeh SD, Larson SM, Burch L, et al. Radioimmunodetection of neuroblastoma with iodine-1313F8: correlation with biopsy, iodine-131-metaiodobenzylguanidine and standard diagnostic modalities. J Nucl Med. 1991;32(5):769-776. [PubMed: 1902508]

19. Kramer K, Kushner BH, Modak S, et al. Compartmental intrathecal radioimmunotherapy: results for treatment for metastatic CNS neuroblastoma. J Neuro-oncol. 2010;97(3):409-418.

20. Humm JL. Dosimetric aspects of radiolabeled antibodies for tumor therapy. J Nucl Med. 1986;27(9):1490-1497. [PubMed: 3528417]

21. Humm JL, Cobb LM. Nonuniformity of tumor dose in radioimmunotherapy. J Nucl Med. 1990;31(1):75-83. [PubMed: 2295944]

22. Kramer K, Cheung NK, Humm JL, et al. Targeted radioimmunotherapy for leptomeningeal cancer using (131)I-3F8. Med Pediatr Oncol. 2000;35(6):716-718. [PubMed: 11107154]

23. Kadoch C, Li J, Wong VS, et al. Complement activation and intra-ventricular rituximab distribution in recurrent central nervous system lymphoma. Clin Cancer Res. 2014;20(4):1029_ 1041. [PubMed: 24190981]

24. Koschmann C, Bloom K, Upadhyaya S, et al. Survival after relapse of medulloblastoma. J Pediatr Hematol Oncol. 2016;38(4):269-273. [PubMed: 26907655]

25. Shih CS, Hale GA, Gronewold L, et al. High-dose chemotherapy with autologous stem cell rescue for children with recurrent malignant brain tumors. Cancer. 2008;112(6):1345-1353. [PubMed: 18224664]

26. Bakst RL, Dunkel IJ, Gilheeney S, et al. Reirradiation for recurrent medulloblastoma. Cancer. 2011;117(21):4977-4982. [PubMed: 21495027]

27. Robison NJ,Campigotto F, Chi SN, et al. A phase II trial of a multi-agent oral antiangiogenic (metronomic) regimen in children with recurrent or progressive cancer. Pediatr Blood Cancer. 2014;61(4):636-642. [PubMed: 24123865]

28. Dunkel IJ, Gardner SL, Garvin JH Jr., et al. High-dose carboplatin, thiotepa, and etoposide with autologous stem cell rescue for patients with previously irradiated recurrent medulloblastoma. Neuro-oncology. 2010;12(3):297-303. [PubMed: 20167818]

29. Kramer K, Pandit-Taskar N, Zanzonico P, et al. Low incidence of radionecrosis in children treated with conventional radiation therapy and intrathecal radioimmunotherapy. J Neuro-oncol. 2015;123(2):245-249.

30. Kramer K, Cheung NK, Humm J, et al. Pharmacokinetics and acute toxicology of intraventricular 131 I-monoclonal antibody targeting disialo-ganglioside in non-human primates. J Neuro-oncol. 1997;35(2):101-111.

31. Modak S, Kramer K, Gultekin SH, et al. Monoclonal antibody $8 \mathrm{H} 9$ targets a novel cell surface antigen expressed by a wide spectrum of human solid tumors. Cancer Res. 2001;61(10):40484054. [PubMed: 11358824] 


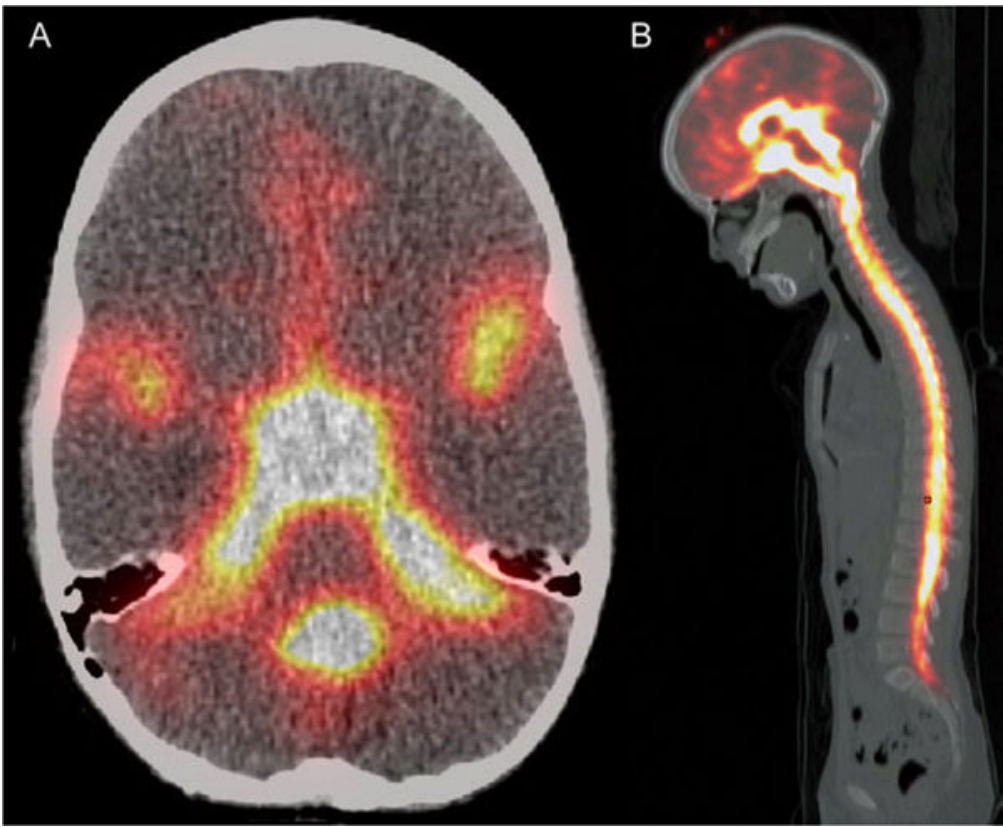

FIGURE 1.

Whole body images of a fused MRI and PET scan following intraventricular injection of radiolabeled antibody (3F8) showing activity in the ventricles (A), subdural space, and spinal canal via sagittal images (B) 
A

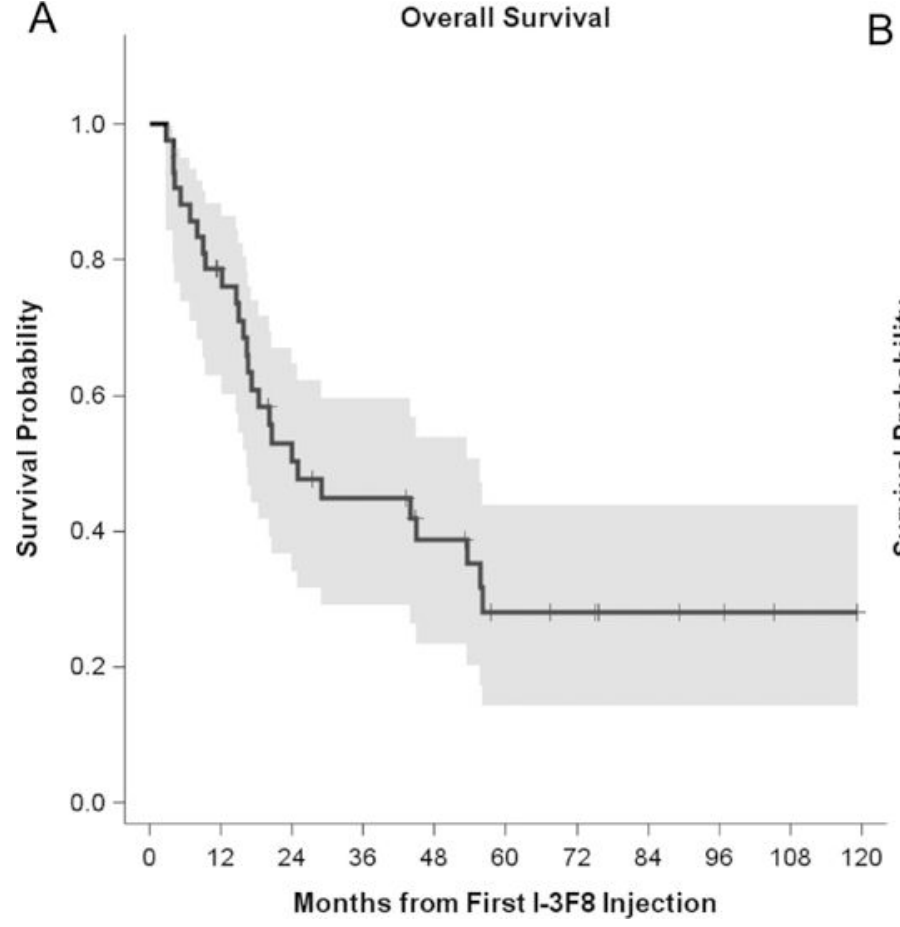

B

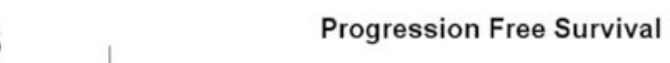

FIGURE 2.

Kaplan-Meier demonstrating overall survival (A) and progression-free survival (B) from the date of first cRIT injection for 42 patients 
TABLE 1

Summary of 42 patients treated for medulloblastoma with cRIT-3F8

\begin{tabular}{|c|c|c|}
\hline Characteristics & & $\mathbf{N}(\%)$ \\
\hline Age at initial diagnosis (years) & Median (range) & $5(1-33)$ \\
\hline Age at first cRIT injection (years) & Median (range) & $7(2-43)$ \\
\hline Histology & Classic & $29(69)$ \\
\hline & Anaplastic & $8(19)$ \\
\hline & PNET & $3(7.1)$ \\
\hline & Desmoplastic & $1(2.4)$ \\
\hline & Medullomyoblastoma & $1(2.4)$ \\
\hline Stage at diagnosis & M0 & $28(66.7)$ \\
\hline & M3 & $14(33.3)$ \\
\hline Craniospinal radiation treatment at initial diagnosis & Yes & $29(69)$ \\
\hline \# Relapses prior to cRIT & 0 & $8(19)$ \\
\hline & 1 & $16(38.1)$ \\
\hline & 2 & $12(28.6)$ \\
\hline & 3 & $3(7.1)$ \\
\hline & 4 & $1(2.4)$ \\
\hline & 5 & $2(4.8)$ \\
\hline Prior treatment with myeloablative chemotherapies prior to cRIT & Yes & $40(95.2)$ \\
\hline Stage at relapse & M0 & $2(4.8)$ \\
\hline & M2 & $5(11.9)$ \\
\hline & $\mathrm{M} 2 / \mathrm{M} 3$ & $1(2.4)$ \\
\hline & M3 & $31(73.8)$ \\
\hline & M3/M4 & $3(7.1)$ \\
\hline Positive CSF cytology at any time & Yes & $19(45.2)$ \\
\hline Status when treated with cRIT & Stable evaluable disease & $19(45.2)$ \\
\hline & Progressive evaluable disease & $3(7.1)$ \\
\hline & Radiographic/cytologic remission & $20(47.6)$ \\
\hline
\end{tabular}

Numbers represent frequencies with percentage in parentheses unless otherwise specified. 


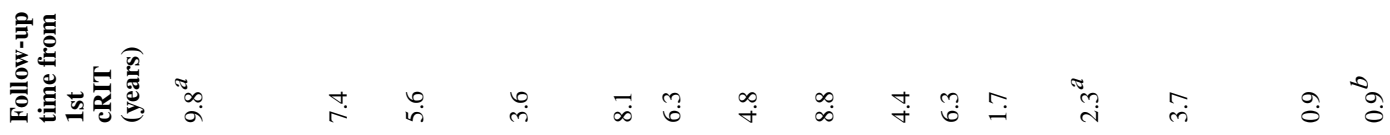

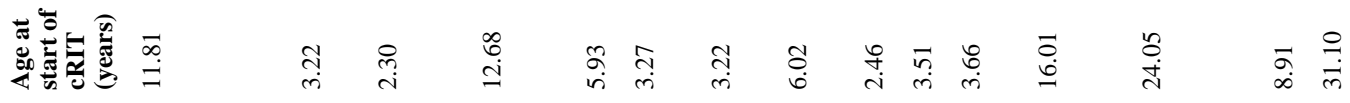
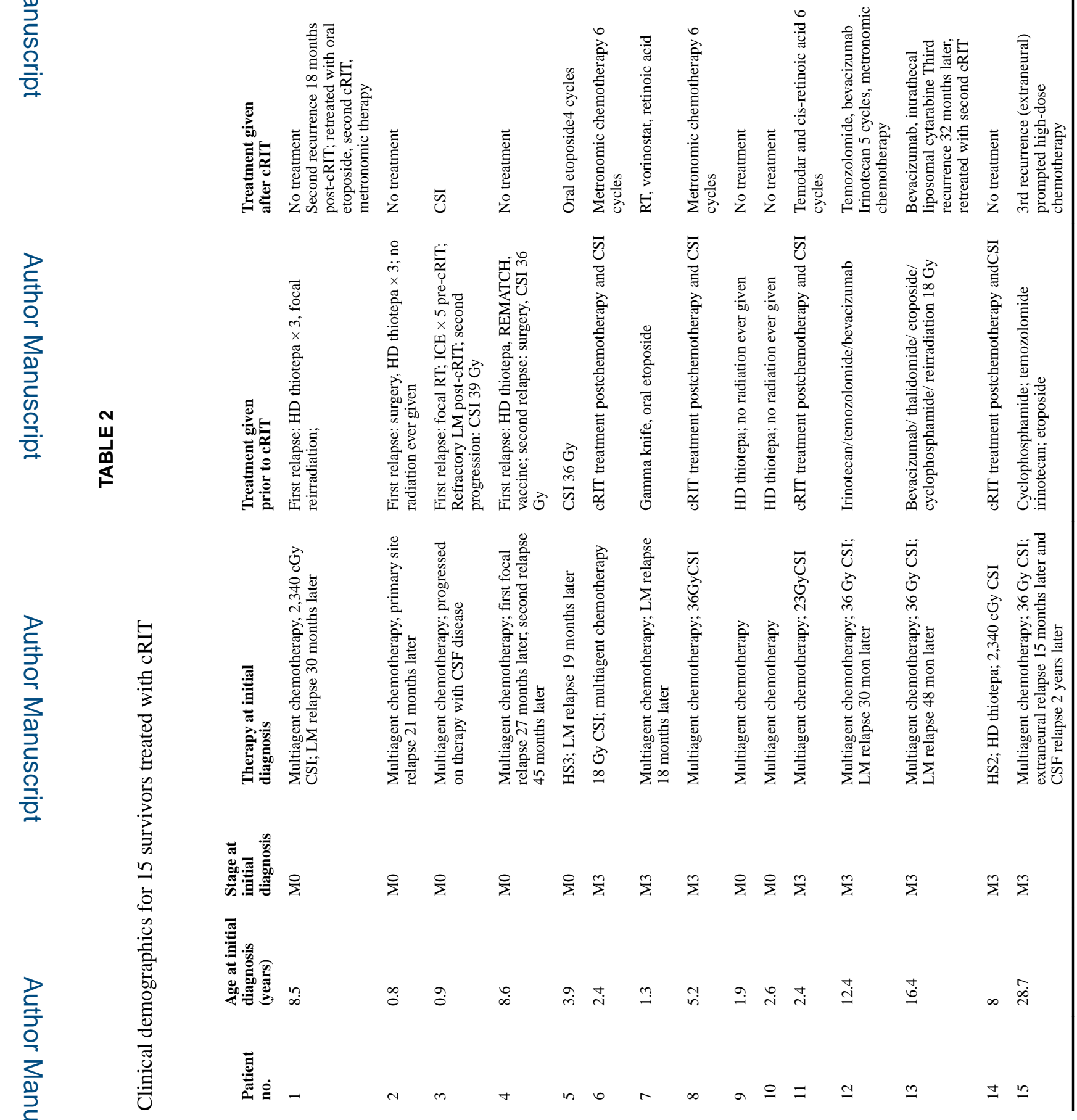

Pediatr Blood Cancer. Author manuscript; available in PMC 2019 August 14. 


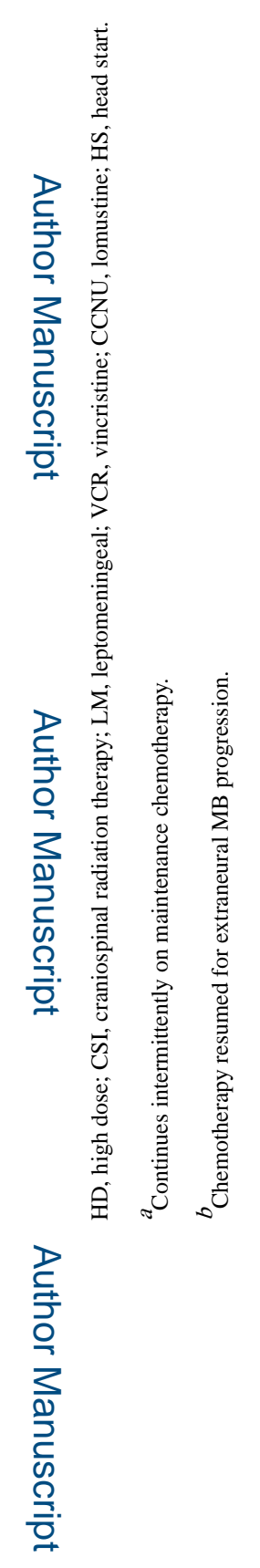

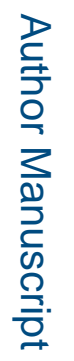

Pediatr Blood Cancer. Author manuscript; available in PMC 2019 August 14. 


\section{TABLE 3}

\section{cRIT results in 42 patients}

$\begin{array}{llc}\text { Characteristics } & & \text { Median (range) or N (\%) } \\ \text { Median CSF dose (cGy/mCi) by sampling } & & 47.9(14.8-207.6) \\ \text { Median blood dose (cGy/mCi) by sampling } & & 1.6(0.1-9.2) \\ \text { Median total CSF dose by sampling (cGy) } & (\mathrm{N}=41) & 1,453(350.0-2,784) \\ \text { Evaluated with } & & 19(45 \%) \\ & { }^{124} \mathrm{I}-3 \mathrm{~F} 8 \text { PET } & 23(55 \%) \\ \text { Median ventricular dose }{ }^{124} \mathrm{I}-3 \mathrm{~F} 8 \text { PET (cGy/mCi) } & (\mathrm{N}=15) & 31.1(13.6-177.0) \\ \text { Median ventricular dose }{ }^{131} \mathrm{I}-3 \mathrm{~F} 8 \mathrm{I} P \mathrm{~F} 8 \mathrm{SPECT}(\mathrm{cGy} / \mathrm{mCi}) & (\mathrm{N}=21) & 89.2(21.7-222.0) \\ \text { Median thoracic spine dose }{ }^{124} \mathrm{I}-3 \mathrm{~F} 8 \text { PET }(\mathrm{cGy} / \mathrm{mCi}) & (\mathrm{N}=17) & 17.2(7.2-57.0) \\ \text { Median thoracic spine dose }{ }^{131} \mathrm{I}-3 \mathrm{~F} 8 \mathrm{SPECT}(\mathrm{cGy} / \mathrm{mCi}) & (\mathrm{N}=22) & 19.6(8.4-89.6) \\ \text { Patients receiving } 4 \text { cRIT injections } & \text { Yes } & 18(43 \%) \\ \text { Post-cRIT response } & \text { Stable } & 25(60 \%) \\ & \begin{array}{l}\text { Progressive } \\ \text { disease }\end{array} & 17(41 \%)\end{array}$

Numbers represent frequencies with percentage in parentheses unless otherwise specified. 


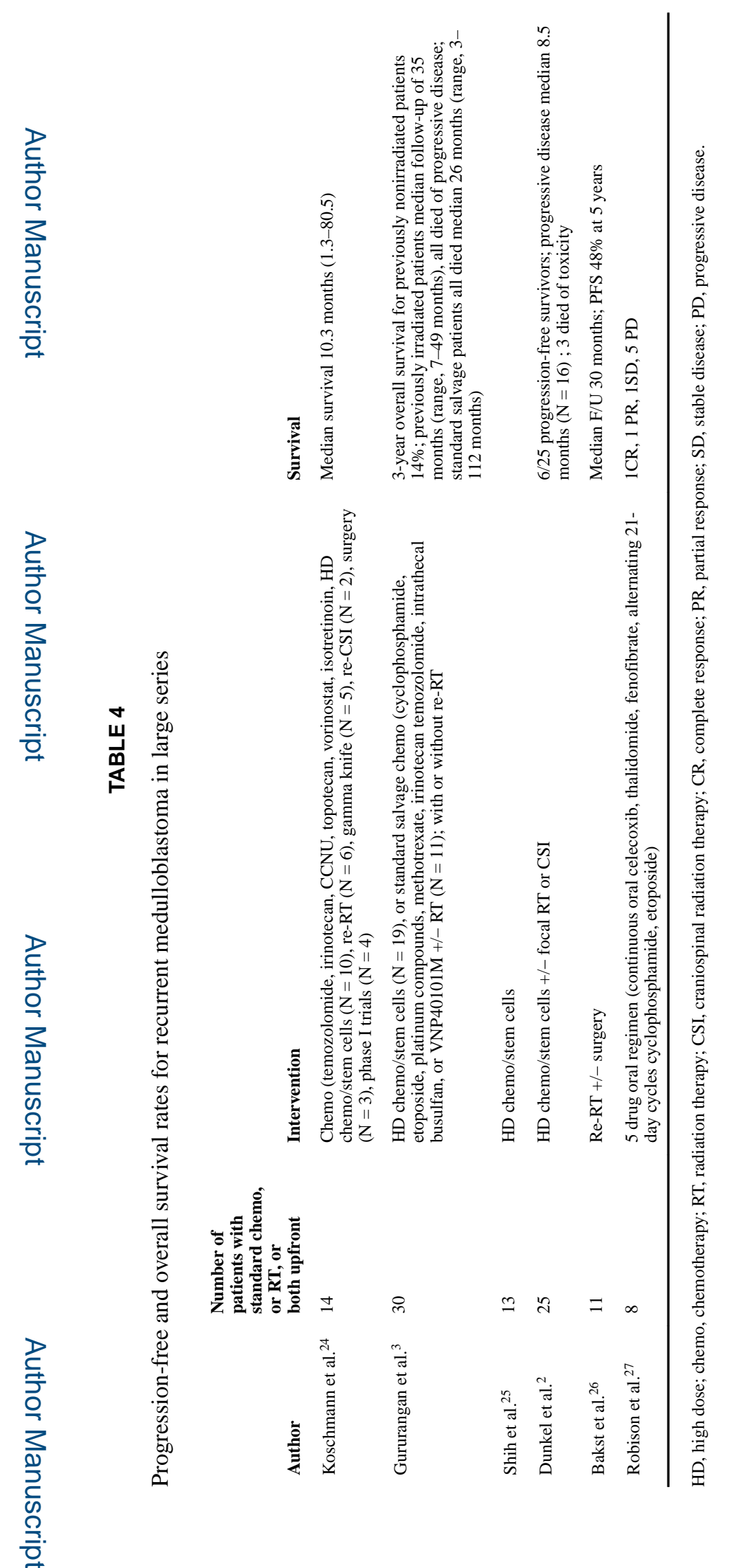

Pediatr Blood Cancer. Author manuscript; available in PMC 2019 August 14. 